\title{
A DIRECT PROOF OF A THEOREM OF WEST ON SEQUENCES OF RIESZ OPERATORS
}

\author{
by ANTHONY F. RUSTON
}

(Received 9 March, 1973)

We recall (cf. [2] Definitions 3.1 and 3.2, p. 322) that a bounded linear operator $T$ on a Banach space $\mathfrak{X}$ into itself is said to be asymptotically quasi-compact if $\kappa\left(T^{n}\right)^{1 / n} \rightarrow 0$ as $n \rightarrow \infty$, where $\kappa(U)=\inf \|U-C\|$ for every bounded linear operator $U$ on $\mathfrak{X}$ into itself, the infimum being taken over all compact linear operators $C$ on $\mathfrak{X}$ into itself. For a complex Banach space, this is equivalent (cf. [2], pp. 319, 321 and 326) to $T$ being a Riesz operator.

THEOREM 1 (West). If the sequence $\left\{T_{n}\right\}$ of asymptotically quasi-compact bounded linear operators on a Banach space $\mathfrak{X}$ into itself converges to the bounded linear operator $T$ on $\mathfrak{X}$ into itself, and $T$ commutes with each operator $T_{n}$ of the sequence, then $T$ is asymptotically quasicompact.

West's proof (cf. [3], Corollary 4.3, p. 135) depends heavily on Banach algebra theory. The following is a direct proof.

Let $\varepsilon>0$ be given. We first choose $p$ so that $\left\|T_{p}-T\right\|<\varepsilon / 3$, and put $U_{p}=T-T_{p}$ (so that $\left\|U_{p}\right\|<\varepsilon / 3$ ); we shall suppose that $U_{p}$ is not the zero operator, for otherwise $T=T_{p}$ and the conclusion follows immediately. We next choose $q$ so that $\kappa\left(T_{p}^{n}\right)^{1 / n}<\varepsilon / 3$ when $n>q$. Then, since $T_{p}$ commutes with $U_{p}$, we have (provided that $n \geqq q$ )

and so

$$
T^{n}=\left(T_{p}+U_{p}\right)^{n}=\sum_{r=0}^{q}\left(\begin{array}{l}
n \\
r
\end{array}\right) T_{p}^{r} U_{p}^{n-r}+\sum_{r=q+1}^{n}\left(\begin{array}{l}
n \\
r
\end{array}\right) T_{p}^{r} U_{p}^{n-r},
$$

$$
\begin{aligned}
\kappa\left(T^{n}\right) & \leqq \sum_{r=0}^{q}\left(\begin{array}{c}
n \\
r
\end{array}\right)\left\|T_{p}\right\| r\left\|U_{p}\right\|^{n-r}+\sum_{r=q+1}^{n}\left(\begin{array}{c}
n \\
r
\end{array}\right) \kappa\left(T_{p}^{r}\right)\left\|U_{p}\right\|^{n-r} \\
& =\left\|U_{p}\right\|^{n} \sum_{r=0}^{q}\left(\begin{array}{l}
n \\
r
\end{array}\right)\left(\left\|T_{p}\right\| /\left\|U_{p}\right\|\right)^{r}+\sum_{r=q+1}^{n}\left(\begin{array}{l}
n \\
r
\end{array}\right) \kappa\left(T_{p}^{r}\right)\left\|U_{p}\right\|^{n-r} .
\end{aligned}
$$

But $\sum_{r=0}^{q}\left(\begin{array}{l}n \\ r\end{array}\right)\left(\left\|T_{p}\right\| /\left\|U_{p}\right\|\right)^{r}$ is a polynomial in $n$, and so there is a positive constant $k$ such that

$$
\sum_{r=0}^{q}\left(\begin{array}{l}
n \\
r
\end{array}\right)\left(\left\|T_{p}\right\| /\left\|U_{p}\right\|\right)^{r}<k \cdot 2^{n}
$$

when $n$ is sufficiently large. Hence

$$
\begin{aligned}
\kappa\left(T^{n}\right) & <(\varepsilon / 3)^{n} \cdot k \cdot 2^{n}+\sum_{r=q+1}^{n}\left(_{r}^{n}\right)(\varepsilon / 3)^{r}(\varepsilon / 3)^{n-r} \\
& \leqq(k+1)(2 / 3)^{n} \varepsilon^{n}
\end{aligned}
$$

when $n$ is sufficiently large. But $(k+1)(2 / 3)^{n} \rightarrow 0$ as $n \rightarrow \infty$. Hence

$$
\kappa\left(T^{n}\right)<\varepsilon^{n}
$$


and so

$$
\kappa\left(T^{n}\right)^{1 / n}<\varepsilon,
$$

when $n$ is sufficiently large. It follows that $\kappa\left(T^{n}\right)^{1 / n} \rightarrow 0$ as $n \rightarrow \infty$, that is, $T$ is asymptotically quasi-compact.

Note. In a later paper ([4]), West discusses the possibility of expressing a Riesz operator as the sum of a compact linear operator and a quasi-nilpotent bounded linear operator, but he does not mention that such an operator is necessarily a Riesz operator; indeed in a later joint paper ([1], p. 108), he and his co-author use an ad hoc argument to prove a particular case. In fact we have the following theorem.

THEOREM 2. If $C$ is a compact linear operator on a Banach space $\mathfrak{\mathfrak { X }}$ into itself, and $Q$ is a quasi-nilpotent bounded linear operator on $\mathfrak{\mathfrak { X }}$ into itself, then $T=C+Q$ is asymptotically quasicompact.

Proof. If we expand $T^{n}=(C+Q)^{n}$ in the natural way, we obtain a sum of $2^{n}$ terms, each term a product of $n$ factors, each factor either $C$ or $Q$ (since we are not assuming that $C$ and $Q$ commute, we cannot combine terms as in the binomial theorem). One term will be $Q^{n}$; all the other terms will have at least one factor $C$, and so will be compact, and their sum will therefore be compact. Hence

$$
\kappa\left(T^{n}\right)^{1 / n} \leqq\left\|Q^{n}\right\|^{1 / n} \rightarrow 0 \quad \text { as } n \rightarrow \infty,
$$

that is $T$ is asymptotically quasi-compact.

Note added in proof. In fact, the completeness of $\mathfrak{X}$ is not essential in Theorems 1 and 2. They remain true for incomplete spaces provided that the definition of asymptotic quasicompactness is extended to these spaces.

\section{REFERENCES}

1. T. A. Gillespie and T. T. West, A characterisation and two examples of Riesz operators, Glasgow Math. J. 9 (1968), 106-110.

2. A. F. Ruston, Operators with a Fredholm theory, J. London Math. Soc. 29 (1954), 318-326.

3. T. T. West, Riesz operators in Banach spaces, Proc. London Math. Soc. (3) 16 (1966), 131-140. $737-752$.

4. T. T. West, The decomposition of Riesz operators, Proc. London Math. Soc. (3) 16 (1966),

\section{University College of North Wales (Coleg Prifysgol Gogledd Cymru) \\ Bangor, CaERnarvonshire, LL57 2UW, WaLES}

